Pacific Journal of Mathematics

NONASSOCIATIVE ALGEBRAS WITH SCALAR INVOLUTION 


\title{
NONASSOCIATIVE ALGEBRAS WITH SCALAR INVOLUTION
}

\author{
KEVIN MCCRIMMON
}

\begin{abstract}
The classical theory of nondegenerate quadratic forms permitting composition has recently been generalized in several directions: Kunze and Scheinberg considered degenerate forms on alternative algebras over fields of characteristic $\neq 2$; Petersson and Racine briefly considered nondegenerate forms over general rings of scalars; the generalized Cayley-Dickson algebras of dimension $2^{n}$ carry a scalar involution, but are not alternative and do not admit composition for $n>3$. In this paper we study general algebras with scalar involution (where all norms $x x^{*}$ and traces $x+x^{*}$ are scalars) over arbitrary rings of scalars. We locate these among all degree 2 algebras, and derive conditions for them to be flexible, alternative, or composition algebras. We consider Cayley elements and Cayley birepresentations, recovering the results of Kunze and Scheinberg on radicals of norm forms. We also investigate the Cayley-Dickson doubling process for constructing new scalar involutions out of old ones.
\end{abstract}

Throughout, $A$ denotes a unital nonassociative algebra over an arbitrary (unital, commutative, associative) ring of scalars $\Phi$. On occasion pathologies in the module structure of $A$ will cause problems. Without loss of generality (replacing $\Phi$ by $\Phi / A^{\perp}$ ) we will always assume that $\Phi$ acts faithfully on $A$,

$$
\alpha A=0 \Rightarrow \alpha=0
$$

or equivalently that $\Phi$ is unitally faithful,

$$
\alpha 1=0 \Rightarrow \alpha=0
$$

(since if $\alpha$ kills the unit it kills all of $A$ ). In order to insure uniqueness of traces and norms we will sometimes impose a stronger condition (unnecessary for free modules or for $\Phi$ without nilpotent elements) of "unital rigidity" (cf. §2).

As usual, an involution is an anti-automorphism of period 2,

$$
(x y)^{*}=y^{*} x^{*}, \quad x^{* *}=x .
$$

A scalar involution is one for which all norms $x x^{*}$ are scalars in $\Phi 1$, hence by linearization all traces $x^{*}+x^{*}$ are too; by faithfulness $\left(0.1^{\prime}\right)$ these 
scalars are uniquely determined,

$$
\begin{gathered}
x x^{*}=N(x) 1, \\
x+x^{*}=T(x) 1 \quad(T(x)=N(x, 1)),
\end{gathered}
$$

where $N, T$ are quadratic and linear forms on $A$. In general, a quadratic form $Q$ is a map $A \rightarrow \Phi$ which is homogeneous of degree 2, $Q(\alpha x)=$ $\alpha^{2} Q(x)$ for $\alpha \in \Phi$, and such that the polarized form

$$
Q(x, y)=Q(x+y)-Q(x)-Q(y)
$$

is bilinear. $Q$ is nondegenerate if its radical

$$
\operatorname{Rad} Q=\{z \in A \mid Q(z)=Q(z, A)=0\}
$$

vanishes.

An algebra has (generic) degree 2 if there exists a linear trace form $T$ and a quadratic norm form $N$ such that for all $x$ in $A$

$$
\begin{gathered}
x^{2}-T(x) x+N(x) 1=0 \\
T(1)=2, \quad N(1)=1 .
\end{gathered}
$$

Linearization of $(0.6)$ leads to

$$
\left(0.6^{\prime}\right) x \circ y-T(x) y-T(y) x+N(x, y) 1=0 \quad(x \circ y=x y+y x) \text {. }
$$

Note that in the presence of $(0.6)$ and unital faithfulness we have $T(1)=2$ iff $N(1)=1$, and $T$ is linear iff $N$ is quadratic (for the "if" part set $y=1$ in $\left(0.6^{\prime}\right)$ to see $T(x) 1$ is linear in $\left.x\right)$. Setting $y=1$ in $\left(0.6^{\prime}\right)$ and using $T(1)=2$ by $(0.7)$ yields $\{N(x, 1)-T(x)\} 1=0$, so by faithfulness we conclude $T(x)=N(x, 1)$, hence by $(0.7)$

(0.8) $T(x)=N(x, 1), N\left(x^{*}\right)=N(x), T\left(x^{*}\right)=T(x)\left(x^{*}=T(x) 1-x\right)$

$$
N\left(x^{2}\right)=N(x)^{2} .
$$

Commutativity and associativity in a general nonassociative algebra are measured by the commutators and associators

$$
[x, y]=x y-y x \text { and }[x, y, z]=(x y) z-x(y z) .
$$

The commuter and the nuclei of $A$ are defined as

Commuter $\operatorname{Comm}(A)=\{x \mid[x, A]=0\}$,

Left nucleus $N_{l}(A)=\{x \mid[x, A, A]=0\}$,

Middle nucleus $N_{m}(A)=\{x \mid[A, x, A]=0\}$,

Right nucleus $N_{r}(A)=\{x \mid[A, A, x]=0\}$,

Nucleus $N(A)=N_{l}(A) \cap N_{m}(A) \cap N_{r}(A)$,

Center $C(A)=\operatorname{Comm}(A) \cap N(A)$. 
1. Scalar involutions and degree 2 . The existence of a scalar involution is closely tied to the degree 2 nature of $A$ : degree 2 is equivalent to the existence of a scalar "linear" involution, which is an algebra involution only under certain conditions on the norm. The precise connection is given by

1.1. TheOREM. Let $A$ be a unitally faithful algebra. Then there exists a linear map ${ }^{*}$ with $1^{*}=1$ and $x x^{*} \in \Phi 1$ for all $x$ iff $A$ has degree 2 and ${ }^{*}$ is its trace involution

$$
x^{*}=T(x) 1-x \quad(T(x)=N(x, 1)) .
$$

Such a map * is always of period 2, but is an algebra involution iff the trace is normal

$$
N(x, y)=T\left(x y^{*}\right) \quad(=T(x) T(y)-T(x y)) .
$$

In this case the trace is commutative,

$$
T(x y)=T(y x),
$$

and conversely if $\Phi$ has no 2-torsion then commutativity implies normality. Thus the algebras with scalar involution are precisely those degree 2 algebras with normal trace (or, if $\Phi$ has no 2-torsion, with commutative trace).

Proof. If ${ }^{*}$ satisfies $x x^{*}=N(x) 1$ as in $(0.3)$ and $1^{*}=1$, then linearization yields $N(1)=1, T(1)=2, x+x^{*}=T(x) 1$ for $T(x)=N(x, 1)$, so $*$ is necessarily the trace involution (1.2). For the trace involution, the scalar condition $x x^{*}=N(x) 1$ of $(0.3)$ is equivalent to the degree 2 condition (0.6) $x^{2}-T(x) x+N(x) 1=0$, and $1^{*}=1$ is equivalent to $T(1)=2$.

The trace involution has period 2 since $T(1)=2$, (or $1^{*}=1$ ). The condition (0.2) that it be an algebra anti-homomorphism can be written as $\left(x y^{*}\right)^{*}=y x^{*}$, and thus holds iff

$$
0=\left(x y^{*}\right)^{*}-y x^{*}=\left\{T\left(x y^{*}\right) 1-x y^{*}\right\}-y x^{*}=\left\{T\left(x y^{*}\right)-N(x, y)\right\} 1
$$

(linearizing (0.3)), hence by faithfulness iff (1.3) holds.

If (1.3) holds then $T(x y)=T(x) T(y)-N(x, y)$ is commutative; conversely, if $T$ is commutative then

$$
\begin{aligned}
2\left\{T\left(x y^{*}\right)-N(x, y)\right\} & =T\left(x y^{*}\right)+T\left(y x^{*}\right)-2 N(x, y) \\
& =T\left(x y^{*}+y x^{*}-N(x, y) 1\right) \quad(\text { by }(0.7))=0
\end{aligned}
$$

(by linearized (0.3)), so if $\Phi$ has no 2-torsion then commutativity (1.4) implies normality (1.3) 
Note that (1.4) is trivial if $A$ itself is commutative, so commutative degree 2 algebras without 2 -torsion always have scalar trace involution. In characteristic 2 situations, commutativity of the trace is not sufficient to imply normality, as the following examples show.

1.5 EXAMPLE. Over any $\Phi$ of characteristic 2 there exist noncommutative degree 2 algebras $A$ with non-normal $T=0$ (trivially such $T$ are commutative!) and $N(x, y) \neq 0$. Indeed, let $A$ be free over $\Phi$ on $1, z, w$ with $z^{2}=w^{2}=0, z w=1, w z=0$. Then $x=\alpha 1+\beta z+\gamma w$ has $x^{2}=$ $N(x) 1$ for $N(x)=\alpha^{2}+\beta \gamma, T(x)=0, N\left(x, x^{\prime}\right)=\beta \gamma^{\prime}+\beta^{\prime} \gamma \neq 0$. Here $x^{*}=x$ is not an algebra involution since $A$ is not commutative. (Note if $A$ is commutative and traceless, $T=0$, then $x^{2}=N(x) 1, N(x, y) 1=x \circ y$ $=2 x y=0$ so $N(x, y)=0$ and $T$ is trivially normal and $x^{*}=x$ is trivially an involution).

1.6. ExAmple. Over any $\Phi$ of characteristic 2 there exists a noncommutative degree 2 algebra $A$ with nonzero non-normal commutative trace. Indeed, let $A$ be free on $1, e, z$ where $e^{2}=e, z^{2}=0, e z=z+e$, $z e=e ;$ then $A$ is degree 2 with respect to the nonzero $T(x)=\beta, N(x)=$ $\alpha(\alpha+\beta), N\left(x, x^{\prime}\right)=\alpha \beta^{\prime}+\alpha^{\prime} \beta$ for $x=\alpha 1+\beta e+\gamma z$. $T$ is commutative since $T([x, y])=T(x \circ y) \in T(\Phi z)=0$, but $T$ is not normal since $N(e, z)=0, T\left(e z^{*}\right)=T(e z)=T(z+e)=1$. (Again we cannot give an example where $A$ is commutative but some $T(e)=1$ : then by $\left(0.6^{\prime}\right)$ $T(x) e+T(e) x+N(x, e)=x \circ e=2 x e=0$ for all $x$ shows $A=\Phi 1 \oplus$ $\Phi e$, and for $x=\alpha 1+\beta e, y=\gamma 1+\delta e$ we have $T(x)=\beta, N(x)=\alpha^{2}+$ $\alpha \beta+\beta^{2} N(e)$, and $N(x, y)=\alpha \delta+\beta \gamma=T\left(x y^{*}\right)$.)

For constructing further examples it will be convenient to analyze algebras $A$ which arise as unital hulls by adjoining a unit to an algebra $A$.

1.7. Proposition. Suppose $A=\Phi 1 \oplus A_{0}$ for an ideal $A_{0} \triangleleft A$.

(i) $A$ has degree iff $x_{0}^{2}=T_{0}\left(x_{0}\right) x_{0}$ for all $x_{0} \in A_{0}$ and some linear functional $T_{0}: A_{0} \rightarrow \Phi\left(\right.$ then $T\left(\alpha 1+x_{0}\right)=2 \alpha+T_{0}\left(x_{0}\right), N\left(\alpha 1+x_{0}\right)=$ $\left.\alpha\left(\alpha+T_{0}\left(x_{0}\right)\right)\right)$;

(ii) When $A$ has degree 2 its norm permits composition $N(x y)=$ $N(x) N(y)$ iff $T_{0}$ permits composition $T_{0}\left(x_{0} y_{0}\right)=T_{0}\left(x_{0}\right) T_{0}\left(y_{0}\right)$ iff $*$ is an algebra involution (and if $\Phi$ has no 2-torsion this holds iff $T_{0}$ is commutative; in particular, any commutative degree 2 hull necessarily has scalar involution and norm permitting composition).

(iii) If $x_{0} y_{0}=s\left(x_{0}, y_{0}\right)$ is an alternating bilinear product on $A_{0}$ and $T_{0}$ : $A_{0} \rightarrow \Phi$ a linear functional such that $T_{0}\left(A_{0}\right) A_{0}=0$, then $A$ has degree 2, and has scalar trace involution iff $T_{0}\left(s\left(A_{0}, A_{0}\right)\right)=0$. 
Proof. (i) is a direct calculation from $A_{0}^{2} \subset A_{0}$. (ii) This follows from (1.1) and direct calculation

$$
\begin{aligned}
N(x y)-N(x) N(y)=\alpha \beta\left\{T_{0}\left(x_{0} y_{0}\right)-\right. & \left.T_{0}\left(x_{0}\right) T_{0}(y)\right\} \\
=\alpha \beta\left\{N(x, y)-T\left(x y^{*}\right)\right\} & \left(x=\alpha 1+x_{0}, y=\beta 1+y_{0}\right) .
\end{aligned}
$$

(iii) We have $x_{0}^{2}=s\left(x_{0}, x_{0}\right)=0=T_{0}\left(x_{0}\right) x_{0}$ by alternation of $s$ and annihiliation of $T_{0}$, hence

$$
T_{0}\left(A_{0}\right) T_{0}\left(A_{0}\right)=T_{0}\left(T_{0}\left(A_{0}\right) A_{0}\right)=0
$$

and

$$
T_{0}\left(x_{0} y_{0}\right)-T_{0}\left(x_{0}\right) T_{0}\left(y_{0}\right)=T\left(s\left(x_{0}, y_{0}\right)\right) \text {. }
$$

2. Unital rigidity. We now investigate conditions under which the trace and norm of a degree 2 algebra are uniquely determined.

2.1. Proposition. If $A$ is a degree 2 algebra with respect to $T, N$ then the other possible $T^{\prime}, N^{\prime}$ satisfying $(0.6-7)$ are precisely all $T^{\prime}=T+F$, $N^{\prime}=N+G$ for linear forms $F$ and quadratic forms $G$ satisfying

$$
\begin{gathered}
F(x) x=G(x) 1 \quad \text { for all } x \in A, \\
F(1)=G(1)=0 .
\end{gathered}
$$

We call such a pair $(F, G)$ satisfying (2.2-3) a compressing pair, and say $A$ is unitally rigid if it has no compressing pairs. In the presence of faithfulness this is just the condition that $A$ cannot be linearly compressed into $\Phi 1$,

$\left(2.2^{\prime}\right) \quad F(x) x \in \Phi 1$ for all $x(F: A \rightarrow \Phi$ linear, $F(1)=0) \Rightarrow F=0$

since by unital faithfulness $F(x) x=G(x) 1$ uniquely defines $G$ making $(F, G)$ a compressing pair.

2.2. Proposition. A degree 2 algebra has a unique trace and norm iff it is unitally rigid.

In most cases unital rigidity implies faithfulness, e.g. if $A$ carries a linear functional $L$ taking on a cancellable value $L(u)=\mu$ in $\Phi$ : if $\alpha A=0$ then $(\alpha L, 0)$ would be compressing, and $\alpha L=0$ would force $\alpha \mu=0$ and hence $\alpha=0$. In particular, this holds if $A$ has degree 2 and $\Phi$ has no 2-torsion, since then $T(1)=2$ is cancellable. But in characteristic 2 rigidity need not imply faithfulness: if $\Phi=\Psi[\varepsilon]$ for $2 \Psi=\varepsilon^{2}=0$ then $A=\Psi$ is a non-faithful $\Phi$-algebra via $\varepsilon A=0$, yet $A=\Phi 1$ is trivially 
unitally rigid and degree 2 under $T(\alpha 1)=0, N(\alpha 1)=\alpha^{2}\left(\alpha=\alpha_{0}+\beta_{0} \varepsilon\right.$ has $\alpha^{2}=\alpha_{0}^{2}, \alpha 1=\alpha_{0}$, so this is well-defined).

Almost any reasonable restriction on $A$ or $\Phi$ guarantees unital rigidity.

2.3. Proposition. A unitally faithful algebra $A$ is unitally rigid if any one of the following conditions hold:

(i) $A=\Phi 1$.

(ii) $\Phi$ has no nilpotent elements.

(iii) $A$ has no trivial ideals $I^{2}=0$.

(iv) $A$ contains a torsion-free element $u(\alpha u=0 \Rightarrow \alpha=0)$ separated from $1(\Phi u \cap \Phi 1=0)$.

(v) $A=\Phi 1 \oplus A_{0}$ where $A_{0}$ contains a torsion-free $u$.

(vi) $A$ is unitally free ( free as $\Phi$-module with basis $\left\{x_{i}\right\}$ where $x_{0}=1$ ).

(vii) $A$ carries a linear map $f$ such that $f(1)=0$ and some $f(u)$ is torsion-free.

(viii) A contains a torsion-free commutator $[u, v]$ or associator $[u, v, w]$.

(ix) $A$ carries a linear form $F$ with $F(1)=0$ and some $F(u)$ is cancellable in $\Phi$.

(x) A has characteristic 2 and carries a quadratic form $Q$ with cancellable trace $Q(u, 1)$ for some $u$.

(xi) $A$ carries a quadratic form $Q$ with $Q(1)=1$ and some $Q\left(u-u^{*}\right)$ cancellable in $\Phi\left(x^{*}=Q(x, 1) 1-x\right)$.

Proof. We must show that if $F, G$ satisfy (2.2-3) then $F=0$ (whence $G(x) 1=0$ forces $G=0$ by faithfulness). (i) is trivial by normalization (2.3). (iii) is a special case of (ii): if $\alpha^{2}=0$ in $\Phi$ then $I=\alpha A$ is trivial ideal in $A$, and $I=0 \Rightarrow \alpha A=0 \Rightarrow \alpha=0$ by faithfulness. (v)-(xi) are all special cases of (iv): clearly (vi) $\Rightarrow$ (v) $\Rightarrow$ (iv), (vii) $\Rightarrow$ (iv) since $u$ is torsion-free if $f(u)$ is, and separated from 1 by $\alpha 1=\beta u \in \Phi 1 \cap \Phi u \Rightarrow$ $\beta f(u)=\alpha f(1)=0 \Rightarrow \beta=0 \Rightarrow \alpha=0$ (by faithfulness), (viii)-(xi) are special cases of (vii) (in (viii) take $f(x)=[x, v]$ or $[x, v, w]$, in (ix) $f(x)=F(x) 1,(\mathrm{x}) \Rightarrow$ (ix) under $F(x)=Q(x, 1),(\mathrm{xi}) \Rightarrow$ (ix) under $F(x)=$ $Q(x, 2 u-Q(u, 1) 1)$ since $F(1)=2 Q(1, u)-2 Q(u, 1) Q(1)=0$ if $Q(1)=$ 1 and $F(u)=4 Q(u)-Q(u, 1)^{2}=4 Q(u)+Q(u, 1)^{2} Q(1)-$ $\left.2 Q(u, 1) Q(u, 1)=Q(2 u-Q(u, 1) 1)=Q\left(u-u^{*}\right)\right)$.

Thus it suffices to verify (ii) and (iv). Linearization of (2.2) yields

$$
F(x) y+F(y) x=G(x, y) 1
$$

and in particular for $y=1 F(x) 1+0=G(x, 1) 1$, so by faithfulness

$$
F(x)=G(x, 1) \text {. }
$$


In case (iv)

$$
F(u) u \in \Phi u \cap \Phi 1=0 \Rightarrow F(u)=0
$$

by torsion-freeness of $u$, hence

$$
\begin{aligned}
F(x) u & =F(x) u+F(u) x \in \Phi 1 \cap \Phi u=0 \quad \text { (by (2.4)) } \\
& \Rightarrow F(x)=0
\end{aligned}
$$

for any $x$, so $F=0$. In case (ii) it suffices to verify $F(x)$ is nilpotent,

$$
F(x)^{2}=F(x) G(x)=G(x)^{2}=0
$$

for all $x$. Applying $F(\cdot), G(x, \cdot)$ to (2.2) yields (via (2.5)) $F(x)^{2}=0$, $2 F(x) G(x)=G(x) F(x)$, so $F^{2}=F G=0$; but then $G(x)^{2} 1=$ $G(x) F(x) x=0$ implies $G^{2}=0$ by faithfulness.

2.7. RemArK. The values of $F, G$ generate a trivial ideal in $\Phi$ (at least when $\Phi$ has no 2-torsion), since

$$
2 F(x) F(y)=2 G(x) F(y)=2 G(x) G(y)=0 .
$$

Indeed, applying $F$ to (2.4) gives $2 F(x) F(y)=0$, hence multiplying (2.2) by $2 F(y)$ gives $2 G(x) F(y)=0$ by faithfulness, then multiplying (2.2) by $2 G(y)$ gives $2 G(y) G(x)=0$ again by faithfulness.

It is easy to exhibit non-rigid degree 2 algebras over arbitrary $\Phi$ containing nilpotent elements.

2.8. EXAMPLE. If $\varepsilon \neq 0=\varepsilon^{2}$ in $\Phi$ then by (1.7) the split null extension $A=\Phi \oplus \Phi \varepsilon$ is a commutative associative degree 2 algebra with scalar involution $T_{0}=0, T(\alpha \oplus \beta \varepsilon)=2 \alpha, N(\alpha \oplus \beta \varepsilon)=\alpha^{2}$ permitting composition, yet $A$ is not unitally rigid since $\varepsilon^{2}=0$ shows $F(\alpha \oplus \beta \varepsilon)=\beta \varepsilon$, $G(\alpha \oplus \beta \varepsilon)=\alpha \beta \varepsilon$ form a nonzero compressing pair.

Over certain rings we can even construct non-rigid commutative associative algebras with nondegenerate norm forms permitting composition.

2.9. ExAmple. For any ideal $\Phi_{0} \triangleleft \Phi$ we obtain by (1.7) a unitally faithful commutative associative $\Phi$-algebra $A=\Phi \oplus \Phi_{0}$ with scalar involution whose norm permits composition via $x y=\alpha \beta \oplus\left(\alpha \beta_{0}+\alpha_{0} \beta+\right.$ $\left.\alpha_{0} \beta_{0}\right)$ for $x=\alpha \oplus \alpha_{0}, y=\beta \oplus \beta_{0}, T_{0}\left(\alpha_{0}\right)=\alpha_{0}, T(x)=2 \alpha+\alpha_{0}, N(x)$ $=\alpha\left(\alpha+\alpha_{0}\right), N(x, y)=2 \alpha \beta+\alpha \beta_{0}+\alpha_{0} \beta$. The norm will be nondegenerate iff

$$
\alpha \in \Phi, 2 \alpha \in \Phi_{0}, \alpha^{2}=\alpha \Phi_{0}=0 \Rightarrow \alpha=0
$$


since $x=\alpha \oplus \alpha_{0} \in \operatorname{Rad} N($ as in $(0.5)) \Leftrightarrow N(x, 1)=N\left(x, \Phi_{0}\right)=N(x)=$ $0 \Leftrightarrow 2 \alpha+\alpha_{0}=\alpha \Phi_{0}=\alpha^{2}=0$. We will have a nonzero compressing map (2.2') $F(x) x \in \Phi 1, F(1)=0$ iff there exists a nonzero $\Phi$-linear map $F_{0}$ : $\Phi_{0} \rightarrow \Phi$ such that

$$
F_{0}\left(\alpha_{0}\right) \alpha_{0}=0 \text { for all } \alpha_{0} \in \Phi_{0} .
$$

First we construct examples where $\Phi$ has no 2-torsion. Let $\Psi$ be an integral domain with no 2-torsion, and let $\Phi=\Psi[\varepsilon, \tau]$ be a polynomial ring in $\tau$ with $\varepsilon^{2}=\varepsilon \tau=0$. Thus $\Phi=\Psi 1 \oplus \Omega_{0} \oplus \Phi_{0}$ is a free $\psi$-module where $\Omega_{0}=\Phi \varepsilon=\Psi \varepsilon, \Phi_{0}=\Phi \tau=\tau \Psi[\tau]$ are ideals in $\Phi$ with $\Omega_{0} \Omega_{0}=$ $\Omega_{0} \Phi_{0}=0$. We have a nonzero $\Phi$-linear map $F_{0}: \Phi_{0} \rightarrow \Omega_{0}$ by $F_{0}(\alpha \tau)=\alpha \varepsilon$ (which is well-defined since $\alpha \tau=0 \Rightarrow \alpha \in \Phi \varepsilon \Rightarrow \alpha \varepsilon=0$ ). Clearly (2.11) holds, $F_{0}\left(\Phi_{0}\right) \Phi_{0} \subset \Omega_{0} \Phi_{0}=0$, so $A=\Phi \oplus \Phi_{0}$ is not unitally rigid. Yet (2.10) holds since $2 \alpha \in \Phi_{0} \cap \Phi_{0}^{\perp}=0\left(\Phi_{0} \subset \Psi[\tau]\right.$ is an integral domain if $\Psi$ is) forces $\alpha=0$ ( $\Phi$ is 2 torsion-free if $\Psi$ is), so the norm is nondegenerate.

In characteristic 2 situations the nondegeneracy condition (2.10) reduces to $\alpha^{2}=\alpha \Phi_{0}=0 \Rightarrow \alpha=0$, so we must make $\Phi_{0}$ sit nicely in all of $\Phi$ (not just in $\Phi_{0}$ ). Let $\Phi=\Lambda(M)$ be the exterior algebra on a free $\Psi$-module $M$ with basis $\left\{x_{i}\right\}$; when $2 \Psi=0$ such $\Phi=\Psi 1 \oplus \Phi_{0}$ is commutative with $\alpha_{0}^{2}=0$ for all $\alpha_{0} \in \Phi_{0} \triangleleft \Phi$, where $\Phi_{0}$ is free over $\Psi$ with basis of all $x_{l_{1}} \wedge \cdots \wedge x_{t_{r}}\left(i_{1}<\cdots<i_{r}, r \geq 1\right)$. If $M$ has infinite rank then $\Phi_{0}^{\perp}=0$ (if $\alpha$ involves $x_{1}, \ldots, x_{n}$ then $x_{n+1} \in \Phi_{0}$, and $\alpha x_{n+1}=0 \Rightarrow \alpha$ $=0$ ), so (2.10) holds and the norm is nondegenerate. Yet (2.11) holds for the imbedding $F_{0}\left(\alpha_{0}\right)=\alpha_{0}$ of $\Phi_{0}$ in $\Phi$, so $A$ is not unitally rigid.

3. Flexible degree 2 algebras. An algebra is flexible if for each $x$ the multiplication operators $L_{x}, R_{x}$ commute: $(x y) x=x(y x)$ for $x$ and $y$,

$$
[x, y, x]=0 \text {. }
$$

All commutative algebras are flexible. A noncommutative Jordan algebra is one such that for each $x$ the multiplication operators $L_{x}, R_{x}, L_{x^{2}}, R_{x^{2}}$ all commute strictly, equivalently the identities

$$
[x, y, x]=\left[x^{2}, y, x\right]=0
$$

hold strictly $(=$ hold in all scalar extensions $=$ their linearizations hold in A). A commutative Jordan algebra is one where $L_{x}=R_{x}$ strictly commutes with $L_{x^{2}}=R_{x^{2}}$ for each $x$,

$$
[x, y]=\left[x^{2}, y, x\right]=0 .
$$


Since $x^{2} \in \Phi x+\Phi 1$ in a degree 2 algebra by (0.6), we see

3.3 Proposition. A degree 2 algebra is a noncommutative Jordan algebra iff it is flexible, and a commutative Jordan algebra iff it is commutative. Any degree 2 algebra $A$ over $\Phi \ni \frac{1}{2}$ has $A^{+}$(under $x \cdot y=\frac{1}{2} x \circ y$ ) the Jordan algebra of the symmetric bilinear form $B(x, y)=\frac{1}{2} N(x, y)$ with basepoint 1:

$$
x \cdot y=B(x, 1) y+B(y, 1) x-B(x, y) 1 .
$$

Flexibility is closely related to a linearized version of composition $N(x y)=N(x) N(y)$, namely the linearization $y \rightarrow y, 1, N(x y, x)=$ $N(x) N(y, 1)$. If $A$ is a unitally faithful degree 2 algebra with norm $N$, trace $T$, and trace involution ${ }^{*}$ then its norm is associative if it satisfies any of the following equivalent conditions:

$$
\begin{aligned}
& N(x y, x)=N(x) T(y) \\
& N(y x, x)=N(x) T(y) \\
& N(x y, z)=N\left(x, z y^{*}\right) \\
& N(x y, z)=N\left(y, x^{*} z\right)
\end{aligned}
$$

the trace is normal, $N(x, y)=T\left(x y^{*}\right)$, and associative,

$$
T((x y) z)=T(x(y z)) .
$$

If $\Phi$ has no 2-torsion these are equivalent to

(3.4iv) the trace is commutative and associative,

$$
T([x, y])=T([x, y, z])=0 \text {. }
$$

(By symmetry it suffices to show (i) $\Rightarrow$ (ii) $\Rightarrow$ (iii) $\Rightarrow$ (i'). Here (i) $\Rightarrow$ (ii) by linearizing $x \rightarrow x, z$; (ii) $\Rightarrow$ normality of the trace by setting $x=1$, hence * is an algebra involution by (1.3), so (ii) $\Rightarrow$ associativity of the trace by $T((x y) z)=N\left(x y, z^{*}\right)=N\left(x, z^{*} y^{*}\right)=N\left(x,(y z)^{*}\right)=T(x(y z))$; (iii) $\Rightarrow$ (i') by $N(y x, x)=T\left((y x) x^{*}\right)=T\left(y\left(x x^{*}\right)\right)=N(x) T(y)$. If $\Phi$ has no 2-torsion we saw in (1.4) that normality is equivalent to commutativity of the trace.) Note that normality is (3.4iii) shows associativity of the norm implies * is a scalar involution.

3.5 THEOREM. A unitally faithful degree 2 algebra is flexible iff

$$
F(x, y) x=G(x, y) 1 \text { for all } x, y
$$

where $F(x, y)=N(x, y)-T\left(x y^{*}\right)$ measures normality and $G(x, y)=$ $N(x y, x)-N(x) T(y)$ measures associativity. Associativity of the norm is 
sufficient for flexibility, and is necessary for flexibility if the algebra is unitally rigid or if ${ }^{*}$ is a scalar involution. Thus an algebra with scalar involution is flexible iff its norm is associative iff its trace is associative.

Proof. In any degree 2 algebra the flexibility condition (3.1) becomes, by $\left(0.6^{\prime}\right)$,

$$
\begin{aligned}
0= & (x y) x-x(y x)=x y \circ x-x(x \circ y) \\
= & \{T(x y) x+T(x) x y-N(x y, x) 1\} \\
& -x\{T(x) y+T(y) x-N(x, y) 1\} \\
= & \{T(x y)-T(y) T(x)+N(x, y)\} x-\{N(x y, x)-T(y) N(x)\} 1 \\
= & F(x, y) x-G(x, y) 1 .
\end{aligned}
$$

If (3.4) holds then $F=G=0$ and $A$ is flexible. If $*$ is an involution then $F=0$ by (1.3), so flexibility holds $\Leftrightarrow G(x, y) 1=0 \Leftrightarrow G=0$ (by faithfulness) $\Leftrightarrow$ (3.4) holds (and (3.4) $=(3.4 i i i)$ reduces to associativity of the trace). If $A$ is unitally rigid as in (2.2-3) then $F x=G 1 \Leftrightarrow F=G=0$ (note $F_{y}(x)=F(x, y), G_{y}(x)=G(x, y)$ have $F_{y}(1)=N(1, y)-T\left(y^{*}\right)$ $=0$ and $G_{y}(1)=N(y, 1)-T(y)=0$ by $\left.(0.8)\right)$, so again (3.4) holds.

3.6. REMARK. If $A$ has a scalar involution then

$$
\begin{aligned}
T([x, y, z]) 1 & =[x, y, z]+[x, y, z]^{*} \\
& =[x, y, z]-\left[z^{*}, y^{*}, x^{*}\right]=[x, y, z]+[z, y, x]
\end{aligned}
$$

(as $a^{*} \equiv-a \bmod \Phi 1$ ), so $A$ is flexible $\Leftrightarrow T$ is associative $\Leftrightarrow A$ satisfies the linearized flexible law $\left(3.1^{\prime}\right)[x, y, z]+[z, y, x]=0$. Thus for algebras with scalar involution, linearized flexibility implies flexibility, even in characteristic 2.

If the norm permits composition it is automatically associative. In the next section we will give examples of flexible degree 2 algebras whose norm is not associative and does not permit composition.

An algebra is left (resp. right) alternative if $x^{2} y=x(x y)$ (resp. $y x^{2}=$ $(y x) x)$, i.e.

$$
\begin{aligned}
& {[x, x, y]=0} \\
& {[y, x, x]=0}
\end{aligned}
$$

and is alternative if it is both left and right alternative. Motivated by the Jordan algebra $J(N, 1)$ of a quadratic form with basepoint, we define

$$
U_{x} y=N\left(x, y^{*}\right) x-N(x) y^{*} .
$$


3.9 Proposition. A degree 2 algebra is left alternative iff $x(y x)=U_{x} y$, right alternative iff $(x y) x=U_{x} y$, and alternative iff it is flexible and $x y x=U_{x} y$. Always $2 U_{x} y=x \circ(x \circ y)-x^{2} \circ y$, so if $\frac{1}{2} \in \Phi$ then $U_{x} y=$ $2 x \cdot(x \cdot y)-x^{2} \cdot y$ is the usual $U$-operator of the Jordan algebra $A^{+}$.

Proof. $x(y x)-U_{x} y=x(y x)-x\left\{x^{*} y^{*}+y x\right\}+\left(x x^{*}\right) y^{*}=$ $\left[x, x^{*}, y^{*}\right]=[x, x, y]$ and dually. By $\left(0.6^{\prime}\right)$

$$
\begin{aligned}
x \circ( & x \circ y)-x^{2} \circ y \\
& =x \circ\{T(x) y+T(y) x-N(x, y) 1\}-\{T(x) x-N(x) 1\} \circ y \\
& =2 T(y)\{T(x) x-N(x) 1\}-2 N(x, y) x+2 N(x) y \\
& =2\{T(x) T(y)-N(x, y)\} x-2 N(x)\{T(y) 1-y\} \\
& =2 U_{x} y .
\end{aligned}
$$

In general an algebra with scalar involution need not be flexible: if $A$ is free over $\Phi$ on $1, e, z$ with $e^{*}=1-e, z^{*}=-z, e^{2}=e, z^{2}=\alpha 1$, $z e=1-2 e+(1-\beta) z, e z=-1+2 e+\beta z$ then $*$ is a scalar involution but $[e, z, e]=1$. Nevertheless, there are lots of flexible algebras with scalar involution. E. Becker [1] investigated the flexible division algebras. He has given a generalized Cayley-Dickson construction of a new scalar involution $(a, b)^{*}=\left(a^{*},-b\right)$ on $\mathbf{C}\left(A, \cdot_{1}, \cdot_{2}, \cdot_{3}\right)=A \oplus A$ under $(a, b)(c, d)=\left(a c+d^{*} \cdot{ }_{1} b, d \cdot{ }_{2} a+b \cdot{ }_{2} c^{*}+b \cdot{ }_{3} d\right)$ where ${ }_{i}$ are bilinear products on $A$ satisfying (i) 1 remains the unit for $\cdot_{2}$, (ii) * remains a scalar involution for $\cdot_{1}, a^{*} \cdot{ }_{1} a=Q(a) 1$, (iii) $\cdot_{3}$ is alternating, $a \cdot{ }_{3} a=0$. Here $N(a, b)=N(a)-Q(b), T(a, b)=T(a)$, and when $A$ is unitally rigid $\mathbf{C}$ is flexible iff (iv) $A$ is flexible, (v) $Q\left(a \cdot{ }_{2} b, c\right)=N\left(b, a^{*} \cdot{ }_{1} c\right)$, (vi) $Q\left(a \cdot{ }_{3} b, b\right)=0$ for all $a, b, c$ in $A$. The ordinary Cayley-Dickson process (cf. §6) is the case $a \cdot{ }_{1} b=\mu a b, a \cdot{ }_{2} b=a b, a \cdot{ }_{3} b=0$.

4. Composition algebras. A quadratic form $Q$ permits composition on an algebra $A$ if

$$
\begin{gathered}
Q(x y)=Q(x) Q(y), \\
Q(1)=1 .
\end{gathered}
$$

In many cases (4.1) already implies (4.2). If $\boxplus$ denotes the ring-direct sum we have

4.3. Lemma. If $Q$ satisfies (4.1) then $\Phi=\Phi_{1} \boxplus \Phi_{0}, A=A_{1} \boxplus A_{0}$, $Q=Q_{1} \boxplus Q_{0}$ where $Q_{1}$ permits composition on $A_{1}$ over $\Phi_{1}$ and $Q_{0}=0$ on $A_{0}$ over $\Phi_{0}$. Thus (4.1) implies (4.2) if $Q$ is nondegenerate or $\Phi$ is an integral domain. 
Proof. $Q(1)=\varepsilon_{1}$ has $\varepsilon_{1}^{2}=\varepsilon_{1}$, so $\Phi=\Phi_{1} \boxplus \Phi_{0}$ for $\Phi_{i}=\Phi \varepsilon_{l}, \varepsilon_{0}=1-$ $\varepsilon_{1}, A=A_{1} \boxplus A_{0}$ for $A_{i}=\varepsilon_{i} A=\Phi_{i} A, Q(a)=Q(1 a)=\varepsilon_{1} Q(a)=\varepsilon_{1}^{2} Q(a)$ $=Q\left(\varepsilon_{1} a\right)$ shows $Q\left(a_{1}+a_{0}\right)=Q\left(a_{1}\right)$.

Linearization of (4.1) shows $Q$ is associative as in (3.4i-ii),

$$
\begin{aligned}
Q(x y, z)=Q\left(y, x^{*} z\right)= & Q\left(x, z y^{*}\right) \\
& \left(x^{*}=T(x) 1-x, T(x)=Q(x, 1)\right)
\end{aligned}
$$

and (4.2) shows

$$
Q\left(x^{*}\right)=Q(x), \quad T\left(x^{*}\right)=T(x), \quad T(1)=2
$$

as in (0.8). In particular, if the norm $Q=N$ of a unitally faithful degree 2 algebra permits composition then $N$ is associative (3.4) and * is a scalar involution.

The existence of a nondegenerate quadratic form permitting composition is tantamount to alternativity (3.7).

4.6 THEOREM. If $Q$ is a nondegenerate quadratic form permitting composition on a unitally faithful algebra $A$, then $A$ is alternative of degree 2 and $Q$ is its norm form. Conversely, the norm of any unitally faithful and rigid degree 2 alternative algebra permits composition.

Proof. If $Q$ permits composition then $x\left(x^{*} y\right)-Q(x) y$ lies in $\operatorname{Rad} Q$ as in $(0.5)$ :

$$
\begin{aligned}
& Q\left(x\left(x^{*} y\right)-Q(x) y\right) \\
& \quad=Q(x) Q\left(x^{*}\right) Q(y)+Q(x)^{2} Q(y)-Q(x) Q\left(x\left(x^{*} y\right), y\right) \\
& \quad=2 Q(x) Q\left(x^{*}\right) Q(y)-Q(x) Q\left(x^{*} y, x^{*} y\right) \quad(\text { by }(4.5),(4.4)) \\
& \quad=0
\end{aligned}
$$

and

$$
\begin{gathered}
Q\left(x\left(x^{*} y\right)-Q(x) y, z\right)=Q\left(x^{*} y, x^{*} z\right)-Q(x) Q(y, z) \quad(\text { by }(4.4)) \\
=Q\left(x^{*}\right) Q(y, z)-Q(x) Q(y, z) \quad \text { (by linearized (4.1)) } \\
=0 \quad(\text { by }(4.5)) .
\end{gathered}
$$

Thus if $Q$ is nondegenerate we have

$$
x\left(x^{*} y\right)=Q(x) y .
$$

Setting $y=1$ in this shows $*$ is scalar with norm $N(x)=Q(x)$, and (4.7) becomes $\left[x, x^{*}, y\right]=0$, which is equivalent to left alternativity $[x, x, y]$ $=0$ as in (3.7l) when $x^{*}=T(x) 1-x$. Dually we have right alternativity 
via the involution (or from flexibility, using (3.5) and associativity (4.4) on $Q=N)$. Thus $A$ is alternative with scalar involution.

Conversely, if $A$ is unitally faithful and rigid and alternative (hence flexible) of degree 2 , then by (3.5) its trace involution is a scalar involution, so its norm permits composition by $N(x y) 1=(x y)(x y)^{*}=x y y^{*} x^{*}$ (Artin's Theorem says alternativity is equivalent to the condition that every two elements generate an associative subalgebra $)=N(y) x x^{*}=$ $N(x) N(y) 1$.

Rigidity is necessary for the converse in (4.6): whenever $\Phi$ contains nilpotent elements we can exhibit a non-rigid associative degree 2 algebra whose norm does not permit composition and whose trace involution is not an algebra involution.

4.8. ExAmple. If $\varepsilon \neq 0, \varepsilon^{2}=0$ in $\Phi$ then in (1.7iii) $A=\Phi 1 \oplus A_{0}$ for $A_{0}=\Phi \varepsilon \oplus \Phi \varepsilon \oplus \Phi \varepsilon, \quad T_{0}(\alpha \varepsilon \oplus \beta \varepsilon \oplus \gamma \varepsilon)=\gamma \varepsilon$, $s\left(\alpha \varepsilon \oplus \beta \varepsilon \oplus \gamma \varepsilon, \alpha^{\prime} \varepsilon \oplus \beta^{\prime} \varepsilon \oplus \gamma^{\prime} \varepsilon\right)=0 \oplus 0 \oplus\left(\alpha \beta^{\prime}-\alpha^{\prime} \beta\right) \varepsilon$

is a degree 2 algebra which does not permit composition nor have scalar involution since $T_{0}(s(\varepsilon \oplus 0 \oplus 0,0 \oplus \varepsilon \oplus 0))=T_{0}(0 \oplus 0 \oplus \varepsilon)=\varepsilon \neq 0$, yet $A$ is trivially associative since $A_{0}^{2} \subset 0 \oplus 0 \oplus \Phi \varepsilon, A_{0}^{2} A_{0}=A_{0} A_{0}^{2}=0$, and hence $[A, A, A]=\left[A_{0}, A_{0}, A_{0}\right]=0$.

For certain $\Phi$ of characteristic 2 we can even construct commutative associative degree 2 algebras with nondegenerate norm but non-normal trace (recall by (1.4) this is impossible if $\Phi$ has no 2-torsion).

4.9. EXAMPLE. If $A=\Phi \oplus \Phi_{0}$ as in (1.7) for $\Phi=\Lambda(M)=\Psi 1 \oplus \Phi_{0}$ of characteristic 2 as in (2.9), $T_{0}\left(\alpha_{0}\right)=\alpha_{0}$, then the norm $N(x)=\alpha^{2}+\alpha \alpha_{0}$ is nondegenerate with $T(x)=\alpha_{0}$ for $x=\alpha \oplus \alpha_{0}$. We have a compressing pair $F(x)=\alpha_{0}, G(x)=\alpha \alpha_{0}, G(x, y)=\alpha \beta_{0}+\alpha_{0} \beta, F(x y)=\alpha \beta_{0}+\alpha_{0} \beta$ $+\alpha_{0} \beta_{0}$ for $y=\beta \oplus \beta_{0}$. Then by (2.2-3) $\lambda F, \lambda G$ is again a compressing pair for any $\lambda \in \Phi$, and by (2.1) $A$ continues to be degree 2 with respect to

$$
\begin{aligned}
& T^{\prime}(x)=T(x)+\lambda F(x)=(1+\lambda) \alpha_{0}, \\
& N^{\prime}(x)=N(x)+\lambda G(x)=\alpha^{2}+(1+\lambda) \alpha \alpha_{0} .
\end{aligned}
$$

By (1.7ii), ${ }^{* \prime}$ is a scalar involution iff $N^{\prime}$ permits composition iff $T_{0}^{\prime}\left(\alpha_{0} \beta_{0}\right)$ $=(1+\lambda) \alpha_{0} \beta_{0}$ equals

$$
T_{0}^{\prime}\left(\alpha_{0}\right) T_{0}^{\prime}\left(\beta_{0}\right)=(1+\lambda)^{2} \alpha_{0} \beta_{0}=\left(1+\lambda^{2}\right) \alpha_{0} \beta_{0},
$$


i.e. iff $\left(\lambda+\lambda^{2}\right) \alpha_{0} \beta_{0}=0$ for all $\alpha_{0}, \beta_{0} \in \Phi_{0}$. But if $M$ is free of infinite rank on $\left\{x_{i}\right\}$, then $\lambda=x_{1}, \alpha_{0}=x_{2}, \beta_{0}=x_{3} \in \Phi_{0} \subset \Phi$ have $\lambda^{2}=0$, $\lambda \alpha_{0} \beta_{0}=x_{1} \wedge x_{2} \wedge x_{3} \neq 0$, and $N, N^{\prime}$ are nondegenerate by (2.10), so $A$ is commutative associative of degree 2 with respect to one nondegenerate scalar involution $*$ permitting composition, and at the same time with respect to another nondegenerate ${ }^{* \prime}$ which is not a scalar involution and does not permit composition.

4.10. Remark. These last two examples also provide examples of flexible degree 2 algebras whose norms are not associative as in (3.4).

In the degenerate norm case, composition is weaker than alternativity.

4.11. ThEOREM. The norm of a unitally faithful algebra $A$ with scalar involution permits composition iff

$$
[x, y, x y]+x[y, y, x]=0
$$

for all $x, y \in A$. For this it is sufficient that $A$ be alternative, and necessary that $A$ be flexible.

Proof. (4.2) holds automatically by (0.7), and (4.1) holds iff

$$
\begin{aligned}
0 & =\{N(x) N(y)-N(x y)\} 1 \quad \text { (by faithfulness) } \\
& =x\left\{N(y) x^{*}\right\}-(x y)(x y)^{*} \\
& =x\left\{\left(y y^{*}\right) x^{*}\right\}-(x y)\left(y^{*} x^{*}\right) \quad(\text { by }(0.3),(0.2)) \\
& =x\left[y, y^{*}, x^{*}\right]-\left[x, y, y^{*} x^{*}\right]=x[y, y, x]+[x, y, x y]
\end{aligned}
$$

(since $a^{*} \equiv-a \bmod \Phi 1$ ). (4.12) implies (3.1) by linearizing $y \rightarrow y, 1$, and alternativity implies that both parts of (4.12) vanish by Artin.

A scalar involution with degenerate norm permitting composition need not be alternative: the composition property tells us nothing about the behavior of $\operatorname{Rad} N$.

4.13. ExAmple. If $N$ permits composition on $A$, then for any $A$-bimodule $Z$ with $a z=z a^{*}, z^{2}=0$ for all $z \in Z, a \in A$, the algebra $A^{\prime}=A$ $\oplus Z$ has scalar involution $(a+z)^{*}=a^{*}-z$ whose norm $N^{\prime}(a+z)=$ $N(a)$ permits composition, yet $A^{\prime}$ need not be alternative (e.g. let $A=\Phi 1$, $Z=\Phi z_{1} \oplus \Phi z_{2}$ for $z_{1}^{2}=z_{2}^{2}=0, z_{1} z_{2}=z_{2}=-z_{2} z_{1}$, so $z_{1}\left(z_{1} z_{2}\right)=z_{2} \neq 0$ $=z_{1}^{2} z_{2}$.

5. Cayley elements and bimodules. Suppose $M$ is a bimodule for an algebra $A$ with involution, i.e. we have unital bilinear products $A M \subset M$ 
and $M A \subset M$. We say an element $m \in M$ commutes with $A$ if $[a, m]=0$, left-associates with $A$ if $[a, b, m]=0$, and $*$-alternates with $A$ if $[m, a, b]$ $=\left[a^{*}, m, b\right]=\left[a^{*}, b^{*}, m\right]$ for $a, b \in A$. We say $M$ is commutative, leftassociative, or *-alternative as $A$-bimodule if all its elements are.

An element $m$ is a *-element for $A$ if

$$
a m=m a^{*}
$$

for all $a \in A ; m$ is a Cayley element (abbreviated $k$.) if

$$
(a b) m=(a m) b^{*}=b(a m)
$$

for all $a, b \in A$, and is a proper Cayley element (p.k.) if all am remain Cayley. $M$ is a Cayley bimodule if all its elements are Cayley (hence properly Cayley).

5.3. Proposition. Let $m$ be an element of an A-bimodule M. Then

(i) $m$ is proper Cayley $\Rightarrow m$ is Cayley $\Rightarrow m$ is $a *$-element;

(ii) $m$ is $a *$-element $\Leftrightarrow[a, m]=\operatorname{Sk}(a) m \Leftrightarrow[m, a]=m \operatorname{Sk}(a)$;

(iii) $m$ is Cayley $\Leftrightarrow m$ is $a *$-element which *-alternates with $A$;

(iv) $m$ is proper Cayley $\Leftrightarrow m$ is Cayley and $[A, A, A] m=0$;

(v) $m$ is proper Cayley $\Leftrightarrow m$ generates a Cayley bimodule $N=A m=m A$, which becomes an $A-*$-bimodule under $n^{*}=-n$;

(vi) if $m$ is a Cayley element then $[a, b, m]=[a, b] m,[m, b, a]=$ $m[a, b]$

(vii) if $M$ is *-alternative (e.g. if $M$ is alternative and ${ }^{*}$ is a scalar involution) then $m$ is proper Cayley $\Leftrightarrow m$ is a*-element.

Proof. (i) If $m$ is p.k. then $1 m$ is $k$.; if $m$ is $k$. then setting $a=1$ in (5.2) shows $b m=m b^{*}$ for all $b \in A$. (ii) is just the definition, where $S k(a)$ stands for $a-a^{*}$. (iii) If $m$ is a $*$-element then

$$
\begin{aligned}
(a b) m-(a m) b^{*} & =[a, b, m]+a\left(b m-m b^{*}\right)-\left[a, m, b^{*}\right] \\
& =[a, b, m]-\left[a, m, b^{*}\right]
\end{aligned}
$$

and

$$
\begin{aligned}
b(a m)-(a b) m & =b\left(m a^{*}\right)-m\left(b^{*} a^{*}\right) \\
& =-\left[b, m, a^{*}\right]+\left(b m-m b^{*}\right) a^{*}+\left[m, b^{*}, a^{*}\right] \\
& =-\left[b, m, a^{*}\right]+\left[m, b^{*}, a^{*}\right],
\end{aligned}
$$

so (5.2) reduces to *-alternativity of $m$. (iv) If $m$ is $k$. then

$$
\begin{aligned}
& (a b)(c m)=\{c(a b)\} m, \\
& \{a(c m)\} b^{*}=\{(c a) m\} b^{*}=\{(c a) b\} m, \\
& b\{a(c m)\}=b\{(c a) m\}=\{(c a) b\} m,
\end{aligned}
$$


so $\mathrm{cm}$ is $k$. iff $[c, A, A] m=0$. (v) $\Leftarrow$ is clear; for $\Rightarrow$, note that $N=A m=$ $m A$ is an $A$-sub-bimodule by (5.1), (5.2), whose elements are $k$. since $m$ is p.k.; if we set $n^{*}=-n$ then the $*$-bimodule conditions $(\text { an })^{*}=n^{*} a^{*}$, $(n a)^{*}=a^{*} n^{*}$ are equivalent to (5.1). (vi) follows from $a(b m)=(b a) m$, ( $m b) a=\left(b^{*} m\right) a=\left(b^{*} a^{*}\right) m=m(a b)$. (vii) follows from (i)-(iii).

An important source of Cayley bimodules are the radicals of norm forms.

5.4. EXAMPLE. If $A$ has scalar involution then $\operatorname{Rad} N$ consists precisely of all skew $*$-elements $z$ for $A$ with $z^{2}=0$ (since $z+z^{*}=N(z, 1) 1$, $\left.z a^{*}+a z^{*}=N(a, z) 1, z^{2}-N(z, 1) z=N(z) 1\right)$. If $A$ is alternative then $\operatorname{Rad} N$ is a Cayley bimodule consisting of all skew proper Cayley elements $z$ of $A$ with $z^{2}=0$ by (5.3(vii)). If $\Phi$ has no 2-torsion, $\operatorname{Rad} N=\operatorname{Rad} N(\cdot, \cdot)$ consists precisely of all skew *-elements, and in the alternative case precisely all skew proper Cayley elements.

The Cayley conditions have strong consequences about the existence and products of Cayley elements inside some enveloping algebra for $A$ and $M$.

\subsection{THEOREM. Let $A$ be an algebra with involution.}

(i) If $A$ has cancellable associators (e.g. contains a Cayley algebra), then $A$ tolerates no proper Cayley elements; any Cayley bimodule has $M=0$.

(ii) If $A$ has cancellable commutators (e.g. contains a quaternion algebra), then $A$ tolerates no left-associative Cayley elements, any left-associative Cayley bimodule has $M=0$, and any Cayley bimodule $M \subset A$ has $M^{2}=0$.

(iii) If $A$ has cancellable elements $S k(a)$ (e.g. contains $a *$-extension of $\Phi)$, then any commutative Cayley bimodule has $M=0$, any left-associative Cayley bimodule $M \subset A$ has $M^{2}=0$, and any Cayley bimodule $M \subset A$ has $M^{3}=0$.

(iv) In general, if $M \subset A$ is a skew Cayley bimodule then $2 M^{4}=0$, and $2 M^{3}=0$ if $M$ is left-associative and $2 M^{2}=0$ if $M$ is commutative (so if $A$ has no 2-torsion we have $M^{4}=0, M^{3}=0, M^{2}=0$ respectively).

More generally, suppose $M$ is an A-bimodule inside an algebra $E$. Suppose $m_{s}^{*}=-m_{s}$ is skew, $m_{*}$ is a *-element, $m_{k}$ is a Cayley element, and $m_{p}$ is a proper Cayley element for $A$. Then for any distribution of parentheses

(v) If $m_{s}, m_{*}, m_{k} \in A$ then

$$
[A, A, A] m_{p}=[A, A] m_{k} m_{p}=S k(A) m_{*} m_{k} m_{p}=2 m_{s} m_{*} m_{k} m_{p}=0 \text {. }
$$


(vi) If $m_{s}, m_{*} \in A$ and $m_{k}$ left-associates with $A$ then

$$
[A, A] m_{k}=S k(A) m_{*} m_{k}=2 m_{s} m_{*} m_{k}=0 \text {. }
$$

(vii) If $m_{s} \in A$ and $m_{*}$ commutes with $A$ then

$$
\operatorname{Sk}(A) m_{*}=2 m_{s} m_{*}=0 \text {. }
$$

Proof. It suffices to establish (v)-(vii). If $m_{s}$ is skew in $A$ then $2 m_{s}=S k\left(m_{s}\right) \in S k(A)$, if $m_{*}$ is a $*$-element then $S k(A) m_{*}=\left[A, m_{*}\right]$ $=m_{*} S k(A)$ by $(5.3 \mathrm{v})$, if $m_{k}$ is $k$. then $[A, A] m_{k}=\left[A, A, m_{k}\right], m_{k}[A, A]$ $=\left[m_{k}, A, A\right]$ by (5.3vi), if $m_{p}$ is p.k. then $[A, A, A] m_{p}=0$ by (5.3iv). Parentheses are unnecessary in (vi) since $m_{k}$ left-associates, and also in (v) since by (5.2)

$$
\begin{gathered}
{[A, A]\left(m_{k} m_{p}\right)=\left\{m_{k}[A, A]\right\} m_{p}, s\left\{m_{*}\left(m_{k} m_{p}\right)\right\}=\left\{m_{k}\left(m_{*} s\right)\right\} m_{p}} \\
s\left\{\left(m_{*} m_{k}\right) m_{p}\right\}=\left\{\left(m_{*} s\right) m_{k}\right\} m_{p},\left(s m_{*}\right)\left(m_{k} m_{p}\right)=\left\{m_{k}\left(s m_{*}\right)\right\} m_{p}, \\
\left\{s\left(m_{*} m_{k}\right)\right\} m_{p}=\left\{\left(m_{*} s\right) m_{k}\right\} m_{p}
\end{gathered}
$$

where $m_{*} s, s m_{*} \in[A, A],[A, A] m_{k}+m_{k}[A, A] \subset[A, A, A]$ for skew $s$.

Combining this with Example 5.4. we obtain the Kunze-Scheinberg results on the radicals of degenerate composition algebras.

5.6. CoROLlaRY (Kunze-Scheinberg [4]). If $A$ is alternative with scalar involution then

(i) $\operatorname{Rad} N=0$ if $A$ contains a Cayley subalgebra, or if $A$ is associative and contains a quaternion subalgebra, or if $A$ is commutative and contains $a$ *-extension of $\Phi$;

(ii) $(\operatorname{Rad} N)^{2}=0$ is trivial if $A$ contains a quaternion subalgebra, or is associative and contains $a *$-extension of $\Phi$;

(iii) $(\operatorname{Rad} N)^{3}=0$ if $A$ contains $a *$-extension of $\Phi$;

(iv) If $A$ has no 2-torsion then $(\operatorname{Rad} N)^{4}=0$, and $(\operatorname{Rad} N)^{3}=0$ if $A$ is associative, and $(\operatorname{Rad} N)^{2}=0$ if $A$ is commutative.

In characteristic 2 situations $M$ need not be nilpotent (even if $A$ is commutative associative), as the following examples show.

EXAMPLE. If $\Phi$ has characteristic 2 and $A=\Lambda(F)$ is the exterior algebra on a free $\Phi$-module $F$ of infinite rank, then $A$ is commutative 
associative with trivial scalar involution $a^{*}=a, T(a)=0, N(a)=\alpha^{2}$ if $a=\alpha 1+m \in A=\Phi 1 \oplus M$ for $M=\sum_{k=1}^{\infty} \Lambda^{k}(F)$ an ideal with $m^{2}=0$ for all $m$; here $M \subset \operatorname{Rad} N$ (and $M=\operatorname{Rad} N$ if $\Phi$ contains no nilpotent elements). $M$ is trivially a Cayley bimodule for $A$, yet $M^{k} \neq 0$ since if $\left\{x_{i}\right\}$ is a basis for $F$ then $0 \neq x_{1} \wedge \cdots \wedge x_{k} \in M^{k}$ for any $k$.

5.8. EXAMPLE. If $A$ is commutative associative with trivial involution $a^{*}=a$ then all elements are Cayley elements, and any ideal $M \triangleleft A$ is a Cayley bimodule; if in addition $\Phi$ has characteristic 2 then $M$ is trivially skew yet we need not have $M^{k}=0$ for any $k: A=\Phi \boxplus \Phi, M=0 \boxplus \Phi=$ $\Phi e$ for $e^{2}=e$ has $M^{k}=M$ for all $k$.

Cayley bimodules are the same as Cayley birepresentations, pairs $(l, r)$ of maps $A \rightarrow \operatorname{End}_{\Phi}(M)$ of the form $l_{a}=f\left(a^{*}\right), r_{a}=f(a)$ for a homomorphism $A \rightarrow \operatorname{End}_{\Phi}(M)$ (since $l_{a}=r_{a^{*}}$ by (5.1), and $r_{a} r_{b}=r_{a b}$ by $(m b) a$ $=\left(b^{*} m\right) a=\left(b^{*} a^{*}\right) m=m(a b)$ from (5.1), (5.2)). There is a canonical construction of cyclic Cayley bimodules for $A$ : if $K$ is any right ideal of $A$ containing all associators $[A, A, A]$ we define $\operatorname{Cay}(A / K)$ to be the space $\bar{A}=A / K$ with $A$-bimodule structure

$$
\operatorname{Cay}(A / K): a \cdot \bar{b}=\overline{b a}, \quad \bar{b} \cdot a=\overline{b a^{*}} \quad\left(K \triangleleft_{r} A, K \supset[A, A, A]\right) .
$$

The associated birepresentation has $l_{a}=\overline{R_{a}}, r_{a}=\overline{R_{a^{*}}}$; these are well-defined precisely when $K$ is a right ideal, and afford a Cayley birepresentation precisely when $f(a)=r_{a}=\overline{R_{a^{*}}}$ is a homomorphism, which is precisely when $[A, A, A] \subset K$ since

$$
\overline{R_{a^{*}} R_{b^{*}}}=\overline{R_{b^{*} a^{*}}} \Leftrightarrow\left(R_{a^{*}} R_{b^{*}}-R_{b^{*} a^{*}}\right) A=\left[A, b^{*}, a^{*}\right] \subset K .
$$

Clearly $\bar{A}=A \overline{1}$ is cyclic with generator $\overline{1}$. The unique minimal such $K$ is the associator ideal $K_{0}=[A, A, A] A=A[A, A, A]$, and every $\operatorname{Cay}(A / K)$ is a homomorphic image of the universal cyclic Cayley bimodule $\operatorname{Cay}\left(A / K_{0}\right)$. The $\operatorname{Cay}(A / K)$ are in 1-1 correspondence with the right ideals of the associativization $\mathscr{A}(A)=A / K_{0}$, hence with the isomorphism classes of cyclic right modules for the associative algebra $\mathscr{A}(A)$. If $A$ is totally nonassociative in the sense that $K_{0}=A, \mathscr{A}(A)=0$ (e.g. if $A$ contains a unital Cayley subalgebra) then $A$ admits no Cayley bimodules.

5.10. THEOREM. The cyclic Cayley bimodules $M=A m$ for $A$ are, up to isomorphism, precisely, all $\mathrm{Cay}(A / K)$ for right ideals $A \supset K \supset[A, A, A]$.

Proof. We have a linear epimorphism $A \rightarrow M$ via $f(a)=a m$. Here $f(a b)=b f(a)$ by (5.2), so $K=\operatorname{Ker} f$ is a right ideal, and $K \supset[A, A, A]$ since $f([a, b, c])=[a, b, c] m=0$ by (5.3iv). Thus we can form the 
Cayley bimodule $\operatorname{Cay}(A / K)$, and the induced linear isomorphism $\operatorname{Cay}(A / K) \stackrel{\bar{f}}{\rightarrow} M$ is a bimodule isomorphism since

$$
\begin{gathered}
\bar{f}(a \cdot \bar{b})=\bar{f}(\overline{b a})=f(b a)=a f(b)=a \bar{f}(\bar{b}), \\
\bar{f}(\bar{b} \cdot a)=\bar{f}\left(\overline{b a^{*}}\right)=f\left(b a^{*}\right)=a^{*} f(b)=f(b) a=\bar{f}(\bar{b}) a
\end{gathered}
$$

5.11 REMARK. This gives another way of seeing the results (5.5i), (5.6i): if $[A, A, A] \subset K$ contains cancellable elements on $A$ then $K m=0$ $\Rightarrow m=0$.

6. The Cayley-Dickson construction. There is a classical way of generating new algebras with scalar involution out of old ones by adjoining a Cayley element. If $A$ is a unital algebra with involution and $\mu$ a cancellable (or faithful) scalar

$$
\begin{aligned}
& \mu a=0 \Rightarrow a=0 \quad \text { in } A \\
& \mu \alpha=0 \Rightarrow \alpha=0 \quad \text { in } \Phi
\end{aligned}
$$

(note that if $A$ is faithful as in (0.1) then the second condition follows from the first, $\mu \alpha=0 \Rightarrow \mu(\alpha A)=0 \Rightarrow \alpha A=0 \Rightarrow \alpha=0)$ then we can construct a new algebra $A \oplus A$ with involution $(a, b)^{*}=\left(a^{*},-b\right)$ and product $(a, b)(c, d)=\left(a c+\mu b^{*} d, d a+b c^{*}\right)$. Letting $l=(0,1)$ we can write this as

$$
\mathbf{C}(A, \mu)=A \oplus A l,
$$

$$
\begin{aligned}
x y=(a+b l)(c+d l) & =\left(a c+\mu d^{*} b\right)+\left(d a+b c^{*}\right) l, \\
x^{*} & =a^{*}-b l .
\end{aligned}
$$

We say $\mathbf{C}(A, \mu)$ is obtained from $A$ by the Cayley-Dickson construction. Note that $l$ is a Cayley element as in (5.2), and that $A l$ is a Cayley bimodule iff $A$ is associative by (5.3iv). It is easily checked that $*$ is an involution on $\mathbf{C}(A, \mu)$ iff it was an involution on $A$, and that * is scalar on $\mathrm{C}(A, \mu)$ iff it is scalar on $A$, with

$$
N(a+b l)=N(a)-\mu N(b), \quad T(a+b l)=T(a) .
$$

From this and cancellability of $\mu$ we see $N$ is nondegenerate on $\mathbf{C}(A, \mu)$ iff it is nondegenerate on $A$. Thus we have a doubling process for creating new scalar involutions out of old ones. The construction depends on the scalar $\mu$ only up to an invertible square, $\mathbf{C}(A, \mu) \cong \mathbf{C}\left(A, \lambda^{2} \mu\right)$, or more generally up to an invertible nuclear norm, $\mathbf{C}(A, \mu) \cong \mathbf{C}(A, N(v) \mu)$ under $a+b l \rightarrow a+(v b) l$ if $v$ is nuclear and $N(v)$ invertible in $\Phi$. 
If $N(x, y)$ is any bilinear form on $A$, we say a subspace $B$ is self-dual if $N^{\cdot}(x)=N(x, \cdot)$ defines an isomorphism of $B$ onto $B^{*}$ (e.g. if $B$ is finite-dimensional over a field $\Phi$ and $N(x, y)$ is nondegenerate on $B)$. In this case $A=B \oplus B^{\perp}: B \cap B^{\perp}=0$ by injectivity, $B+B^{\perp}=A$ by surjectivity of $N^{\bullet}\left(N(a, \cdot)=N(b, \cdot)\right.$ for some $b \in B$, so $\left.a-b \in B^{\perp}\right)$. If $\mu B=B$ then $B \oplus B l$ is again a self-dual subspace inside $\mathbf{C}(A, \mu)$, since $N(B, B l)=0$ and $N(b l, c l)=\mu N(b, c)$. The strange product of (6.2) is necessary for alternativity: such a doubling process goes on constantly in alternative algebras.

6.5. Jacobson Doubling Theorem. Suppose $A$ is alternative with scalar involution whose norm permits composition. If $B$ is a self-dual subalgebra and $l \in B^{\perp}$ has $N(l)=-\mu$ surjective on $B$, then $A$ contains $a$ self-dual subalgebra $\mathbf{C} \cong \mathbf{C}(B, \mu)$.

Proof. We have $B l \subset B^{\perp}$ by (4.4), so $C=B \oplus B l$ is a subspace which is again self-dual since $\mu B=B$ by hypothesis. Also

$$
b l=0 \Rightarrow 0=N(b l, B l)=\mu N(b, B)=N(b, B) \Rightarrow b \in B \cap B^{\perp}=0,
$$

so $C$ is linearly isomorphic to $\mathbf{C}(B, \mu)$. To prove it is algebra-isomorphic (in particular, is itself a subalgebra), it suffices to establish the rules

$$
c(b l)=(b c) l=(b l) c^{*}, \quad(b l)(c l)=\mu c^{*} b
$$

of (6.2); but

$$
\begin{aligned}
& 0=N(1, l) \Rightarrow l^{*}=-l, \\
& 0=N(b, l)=b l^{*}+l b^{*}=l b^{*}-b l
\end{aligned}
$$

show $l$ is a *-element for $B$ as in (5.1), hence by (5.3viii) is a Cayley element by alternativity, and the first rules hold: furthermore,

$$
(b l)(c l)=\left(l b^{*}\right)(c l)=l\left(b^{*} c\right) l=l^{2}\left(c^{*} b\right)=+\mu c^{*} b
$$

by the Moufang law in $A$.

By repeated application of the Cayley-Dickson construction starting from a $*$-extension $\Omega=\Phi 1 \oplus \Phi \omega\left(\omega+\omega^{*}=1,1-4 N(\omega)\right.$ cancellable $)$ - or even from $\Phi 1$, if $\frac{1}{2} \in \Phi$ - we get a 4-dimensional quaternion algebra, an 8-dimensional octonion or Cayley algebra, and then generalized Cayley-Dickson algebras of dimension $2^{n}(n \geq 4)$. As we shall see below, these latter are no longer alternative and no longer permit composition, but they are flexible with scalar involution, and if $\Phi$ is a field are simple (indeed, have no proper 1-sided ideals). 
The general associator in $\mathbf{C}(A, \mu)$ is given by

$$
\begin{aligned}
& {[x, y, z]=[a+b l, c+d l, e+f l]=g+h l \text { for } } \\
& g= {[a, c, e]+\mu\left\{\left(d^{*} b\right) e-\left(e d^{*}\right) b\right\} } \\
&+\mu\left\{f^{*}(d a)-a\left(f^{*} d\right)\right\}+\mu\left\{f^{*}\left(b c^{*}\right)-\left(c^{*} f^{*}\right) b\right\}, \\
& h=\{f(a c)-(f c) a\}+\left\{(d a) e^{*}-\left(d e^{*}\right) a\right\} \\
&+\left\{\left(b c^{*}\right) e^{*}-b\left(e^{*} c^{*}\right)\right\}+\mu\left\{f\left(d^{*} b\right)-b\left(d^{*} f\right)\right\},
\end{aligned}
$$

and the general commutator by

$$
\begin{aligned}
{[x, y] } & =[a+b l, c+d l]=g+h l & \text { for } \\
g & =[a, c]+\mu\left(d^{*} b-b^{*} d\right), & h=d\left(a-a^{*}\right)+b\left(c^{*}-c\right) .
\end{aligned}
$$

The criteria for $\mathbf{C}$ to inherit algebraic properties from $A$ are given by

6.8. THEOREM. If $\mu \in \Phi$ is cancellable then:

(i) $\mathrm{C}(A, \mu)$ has trivial involution $\Leftrightarrow A$ has trivial involution and $2 A=0$.

(ii) $\mathbf{C}(A, \mu)$ is commutative $\Leftrightarrow A$ is commutative with trivial involution.

(iii) $\mathbf{C}(A, \mu)$ is associative $\Leftrightarrow A$ is commutative and associative.

(iv) $\mathbf{C}(A, \mu)$ is flexible $\Leftrightarrow A$ is flexible, all norms aa* commute with $A$, and $[a, b, c]=\left[a, b^{*}, c^{*}\right]$ for all $a, b, c \in A$ (if ${ }^{*}$ is a scalar involution this happens iff $A$ is flexible).

(v) $\mathrm{C}(A, \mu)$ is alternative $\Leftrightarrow A$ is alternative, all norms aa* commute with $A$, and all $a+\mathrm{T}(a)$ lie in the nucleus of $A$ (if $A$ has no 3-torsion or $3 A=A$ or ${ }^{*}$ is a scalar involution, this happens iff $A$ is associative with norms in the center $)$.

(vi) $\mathbf{C}(A, \mu)$ is simple $\Leftrightarrow A$ is *-simple, but not commutative with trivial involution with $\gamma \in C(A), \mu \gamma^{2}=1$.

(vii) $\mathbf{C}(A, \mu)$ is left-simple $\Leftrightarrow A$ is simple, but has no $m$ with $\mu m m^{*}=1$, $[m, A]=[A, m, A]=0, m(a b)=(m b) a$ for all $a, b \in A$.

The commuter, nuclei, and center of $\mathrm{C}(A, \mu)$ are given by

(viii) $\operatorname{Comm}(\mathbf{C}(A, \mu))=\left\{a+b l \mid a=a^{*}, b=b^{*}\right.$ in $\operatorname{Comm}(A)$ with $b S k(A)=0\}$.

(ix) $N_{l}(\mathbf{C}(A, \mu))=N_{r}(\mathbf{C}(A, \mu))=\left\{a+b l \mid a \in C(A), b \in N_{m}(A) \cap\right.$ $\operatorname{Comm}(A)$ with $c_{1}\left(c_{2} b\right)=\left(c_{2} c_{1}\right) b$ and $\left(b c_{2}\right) c_{1}=b\left(c_{1} c_{2}\right)$ for all $\left.c_{1}, c_{2} \in A\right\}$.

(x) $N_{m}(\mathbf{C}(A, \mu))=\left\{a+b l \mid a \in N_{m}(A) \cap \operatorname{Comm}(A), \quad b \in\right.$ $\operatorname{Comm}(A)$ with $c_{1}\left(c_{2} b\right)=c_{2}\left(c_{1} b\right),\left(b c_{2}\right) c_{1}=\left(b c_{1}\right) c_{2}$ for all $c_{1}$, $\left.c_{2} \in A\right\}$. 
(xi) $N(\mathbf{C}(A, \mu))=\{a+b l \mid a, b \in C(A)$ with $b[A, A]=0\}$.

(xii) $C(\mathbf{C}(A, \mu))=\left\{a+b l \mid a=a^{*}, b=b^{*} \in C(A)\right.$ with $\left.b \operatorname{Sk}(A)=0\right\}$.

Thus if $\operatorname{Comm}(A)=C(A)$ then also $\operatorname{Comm}(\mathbf{C}(A, \mu))=C(\mathbf{C}(A, \mu))$.

Proof. (i) is clear from (6.3). (ii) follows directly from (6.7). (iii) $\Rightarrow$ follows from $[a, b, l]=[a, b] l, \Leftarrow$ from $(6.6)$.

For (iv) we set $e=a, f=b$ in (6.6) and recall that $\mu$ is cancellable to see $[x, y, x]=0$ for all $x, y$ in $\mathbf{C}(A, \mu)$ iff $A$ is flexible,

$$
\begin{aligned}
(d a) a^{*}-\left(d a^{*}\right) a & =0, \\
b^{*}\left(b c^{*}\right)-\left(c^{*} b^{*}\right) b & =0, \\
b(a c)-(b c) a+\left(b c^{*}\right) a^{*}-b\left(a^{*} c^{*}\right) & =0, \\
\left(d^{*} b\right) a-\left(a d^{*}\right) b+b^{*}(d a)-a\left(b^{*} d\right) & =0,
\end{aligned}
$$

which can be rewritten (using flexibility) as:

(iv.1) $A$ is flexible.

(iv.2) $\left[d, a^{*}, a\right]-\left[d, a, a^{*}\right]=d\left[a, a^{*}\right]$.

(iv.3) $\left[c^{*}, b, b^{*}\right]-\left[c^{*}, b^{*}, b\right]=\left[c^{*}, b^{*} b\right]$.

(iv.4) $[b, c, a]-\left[b, c^{*}, a^{*}\right]=b\left\{[a, c]+\left[c^{*}, a^{*}\right]\right\}$.

(iv.5) $\left[a, d, b^{*}\right]-\left[a, d^{*}, b\right]=\left[a, d^{*} b+b^{*} d\right]$.

Linearizing $b \rightarrow b, 1$ in (3) we see $0=\left[c^{*}, \mathrm{~T}(b)\right]$, so all traces $\mathrm{T}(b)$ commute with $A$; thus

$$
\left[c^{*}, a^{*}\right]=[-c,-a]=[c, a]=-[a, c],
$$

so (4) becomes $[b, c, a]=\left[b, c^{*}, a^{*}\right]$; thus all left sides of (2)-(5) vanish, as do the right sides of (2) and (4), while the right side of (5) is a linearization of that of (3), which in turn is just the condition that all norms $b b^{*}$ commute. (If $*$ is scalar then automatically all norms in $\Phi 1$ commute, and $a^{*} \equiv-a \bmod \Phi 1$, so automatically $\left[b, c^{*}, a^{*}\right]=[b, c, a]$, hence in this case flexibility alone suffices).

For (v) we set $c=a, d=b$ in (6.6) to see $[x, x, y]=0$ for all $x, y$ (hence, via the involution, $[y, x, x]=0$ and $\mathbf{C}$ is alternative) iff $A$ is alternative,

$$
\begin{aligned}
\left(b^{*} b\right) e-\left(e b^{*}\right) b & =0, \\
f\left(b^{*} b\right)-b\left(b^{*} f\right) & =0, \\
(b a) e^{*}-\left(b e^{*}\right) a+\left(b a^{*}\right) e^{*}-b\left(e^{*} a^{*}\right) & =0, \\
f^{*}(b a)-a\left(f^{*} b\right)+f^{*}\left(b a^{*}\right)-\left(a^{*} f^{*}\right) b & =0,
\end{aligned}
$$


which can be rewritten (using alternativity, and the fact that by flexibility all norms and traces commute, so $b b^{*}=b^{*} b$ ) as:

(v.1) $A$ is alternative and all norms $a a^{*}$ commute with $A$.

(v.2) $\left[e, b^{*}, b\right]=0$.

(v.3) $\left[b, b^{*}, f\right]=0$.

(v.4) $\left[b, \mathrm{~T}(a)+a, e^{*}\right]=0$.

(v.5) $\left[f^{*}, b, \mathrm{~T}(a)+a\right]=0$.

Here (4)-(5) is the condition that all $a+\mathrm{T}(a)$ lie in the nucleus of $A$, in which case (2)-(3) follow from alternativity $\left(\left[e, b^{*}, b\right]=\left[e, b^{*}+2 b, b\right]\right.$ $=[e, b+\mathrm{T}(b), b]=0$ ). (If * is scalar then $\mathrm{T}(a) \in \Phi 1$ and hence all $a$ are nuclear, $A=N$ is associative; since $\{\mathrm{T}(a)+a\}+\left\{\mathrm{T}\left(a^{*}\right)+a^{*}\right\}=3 \mathrm{~T}(a)$ is nuclear, we see $3 a$ is nuclear, [ $3 A, A, A]=0$, so if $3 A=A$ or if $A$ has no 3-torsion then $[A, A, A]=0$ and again $A$ is associative.)

For (vi), for $\mathbf{C}$ to be simple certainly $A$ must be $*$-simple since if $B \triangleleft A$ were a proper $*$-ideal (6.2) shows $B+B l$ would be a proper ideal in $\mathrm{C}(A, \mu)$. Also $A$ cannot have $*=\mathrm{Id}, \mu \gamma^{2}=1$ since then $A(1+\gamma l)$ is easily checked to be a proper ideal in $\mathrm{C}(A, \mu)$. Now suppose $A$ is *-simple and $\mathscr{D}$ is a proper ideal in C. When $A$ is $*$-simple, $\mu \neq 0$ in $\Phi$ is invertible in the centroid, so $\mu A=A$. From (6.2) and $\mu A=A$ we see $\mathscr{D} \cap A$ is a $*$-ideal in $A$ (note $l(a l)=\mu a^{*}$ ) which does not contain 1 , so $\mathscr{D} \cap A=0$, hence $\mathscr{D} \cap A l=0$ too (since $l(\mathscr{D} \cap A l) \subset \mathscr{D} \cap A=0$ and $l$ is injective). Thus the elements of $\mathscr{D}$ have the form $b+f(b) l=g(c)+c l$ where $f, g$ are inverse bijections between $B=\{b \in A \mid$ some $b+d l \in \mathscr{D}\}$ and $C=\{c \in A \mid$ some $a+c l \in \mathscr{D}\}$. Now from (6.2) $B \triangleleft A, C \triangleleft_{r} A, B+$ $B^{*} \subset C, \mu\left(C+C^{*}\right) \subset B$, so if $\mathscr{D}$ is nonzero we have $B \neq 0$, the $*$-ideal $B+B^{*}$ must be $A$, so $C=A, B \supset \mu A=A$, and $B=A$ too. But (6.2) says $f, g: A \rightarrow A$ satisfy

$$
f(a b)=f(b) a, \quad f(b a)=f(b) a^{*}, \quad g(a b)=\mu a^{*} f(b),
$$

so $f(1)=m$ satisfies $f(a)=m a=m a^{*}, f\left(a-a^{*}\right)=0 \Rightarrow a^{*}=a$ and $A$ is commutative with trivial involution, $m(a b)=(m b) a$ shows $m=\gamma$ is in the center of $A$ (center = left nucleus for commutative algebras) with $1=f(g(1))=f(\mu m)=\mu m^{2}=\mu \gamma^{2}$, and this is precisely the case we ruled out in (vi).

For (vii), for $\mathbf{C}$ to be left-simple certainly $A$ must be simple, since if $B \triangleleft A$ were a proper ideal then (6.2) shows that $B+B^{*} l$ would be a proper left ideal in C. Also, a direct calculation shows that if $m \in A$ satisfies $[a, m]=[a, m, b]=0, \mu m m^{*}=1, m(a b)=(m b) a$ for all $a, b \in A$ then $\mu m\left\{\left(b^{*} m^{*}\right) a\right\}=a b^{*}$ and so $\mathscr{D}=A(1+m l)$ is a proper left ideal in C. Now suppose $A$ is simple, so $\mu \neq 0$ forces $\mu A=A$, and $\mathscr{D}$ is a proper left ideal in C. Then $\mathscr{D} \cap A$ is an ideal in $A$ (clearly left, and right since $(A l)\{l(d)\}=\mu d A=d A$ ) which doesn't contain 1 , so $\mathscr{D} \cap A=0$, hence 
again $\mathscr{D} \cap A l=0$. Once more $\mathscr{D}$ consists of all $b+c l$ where $c=f(b)$, $b=g(c)$ for bijections between $B$ and $C$ as above. From (6.2) $B \triangleleft_{l} A$, $B^{*} \subset C, C^{*} A \subset B$, so $C=B^{*}, B A \subset B$ and $B \triangleleft A$; by simplicity $B=C$ $=A$ if $\mathscr{D} \neq 0$. Again (6.2) says

$$
f(a b)=f(b) a, \quad g\left(a b^{*}\right)=\mu f(b)^{*} a,
$$

so $f(1)=m$ has

$$
\begin{aligned}
& f(a)=m a, \quad m(a b)=(m b) a, \\
& g(a)=\mu m^{*} a=\mu a m^{*}, \quad(a c) m^{*}=\left(c m^{*}\right) a,
\end{aligned}
$$

so

$$
\left[m^{*}, A\right]=0 \Rightarrow[m, A]=0, \quad 1=g(f(1))=g(m)=\mu m m^{*},
$$

$(b m) a=(m b) a=m(a b)=\left\{\left(b^{*} a^{*}\right) m^{*}\right\}^{*}=\left\{\left(a^{*} m^{*}\right) b^{*}\right\}^{*}=b(m a)$, so $[b, m, a]=0$, and $m$ is one of the elements forbidden by (viii).

For (viii)-(xii) recall the definitions (0.10) of commuter and nuclei. (viii) follows immediately from (6.7) (note $d^{*} b=b d=b d^{*} \Rightarrow b \in$ $\operatorname{Comm}(A))$. The sufficiency of the conditions in (ix) $-(\mathrm{x})$ follows easily from (6.6); for necessity note

$$
\begin{aligned}
& a+b l \in N_{\varepsilon}(\mathbf{C}) \Leftrightarrow a, b l \in N_{\varepsilon}(\mathbf{C}) ; \\
& a \in N_{\varepsilon}(\mathbf{C}) \Rightarrow a \in N_{\varepsilon}(A) \text {; } \\
& a \in N_{l}(\mathbf{C}) \Rightarrow 0=-\left[a, c l, d^{*} l\right]=\mu\{[d, c, a]+[a, d c]\} \\
& \Rightarrow a \in \operatorname{Comm}(A) \cap N_{l}(A) \cap N_{r}(A)=C(A) ; \\
& a \in N_{m}(\mathbf{C}) \Rightarrow 0=[c, a, l]=[c, a] l \Rightarrow a \in \operatorname{Comm}(A) \cap N_{m}(A) \text {; } \\
& b l \in N_{l}(\mathbf{C}) \Rightarrow 0=[b l, c l, l]=\mu\left[c^{*}, b\right] l \Rightarrow b \in \operatorname{Comm}(A) \text {; } \\
& 0=\left[b l, c_{1}^{*}, c_{2}^{*}\right]=\left[b l, c_{2}^{*}, c_{1}^{*} l\right] \\
& \Rightarrow \quad 0=\left(b c_{1}\right) c_{2}-b\left(c_{2} c_{1}\right)=c_{1}\left(b c_{2}\right)-\left(c_{2} c_{1}\right) b \\
& \Rightarrow\left(c_{1} b\right) c_{2}=\left(c_{2} c_{1}\right) b=c_{1}\left(b c_{2}\right) \\
& \text { (so } \left.b l \in N_{m}(A)\right) \text { and } c_{1}\left(c_{2} b\right)=\left(c_{2} c_{1}\right) b \text {; }
\end{aligned}
$$

similarly,

$$
b l \in N_{m}(\mathbf{C}) \Rightarrow 0=[c l, b l, l]=\mu\left[b^{*}, c\right] l \Rightarrow b \in \operatorname{Comm}(A),
$$

so

$$
\begin{aligned}
0 & =\left[c_{1}, b l, c_{2}^{*} l\right]=\left[c_{1}^{*} l, b l, c_{2}^{*} l\right] \\
& \Rightarrow 0=c_{2}\left(b c_{1}\right)-c_{1}\left(c_{2} b\right)=c_{2}^{*}\left(b^{*} c_{1}^{*}\right)-c_{1}^{*}\left(b^{*} c_{2}^{*}\right) \\
& \Rightarrow c_{2}\left(c_{1} b\right)=c_{1}\left(c_{2} b\right), \quad\left(b c_{1}\right) c_{2}=\left(b c_{2}\right) c_{1} .
\end{aligned}
$$

(xi) follows by combining (ix) and (x), since for commuting $b \quad c_{2}\left(c_{1} b\right)=$ $c_{1}\left(c_{2} b\right)=\left(c_{2} c_{1}\right) b$ implies $b \in N_{r}(A)$. (xii) follows by combining (viii) and (xi), since $b S k(A)=0 \Rightarrow b[A, A]=0$ for central $b(b[x, y]=b(x y)-$ $\left.b(y x)^{*}=b x^{*} y-b x^{*} y^{*}=x^{*} b\left(y-y^{*}\right)=0\right)$. 
6.9. Corollary. If $A$ is a generalized Cayley-Dickson algebra of dimension $2^{n}$ obtained from $\Phi 1$ (or $a *$-extension) by repeated application of the Cayley-Dickson process, then

(i) $\operatorname{Comm}(A)=C(A)$,

(ii) $C_{*}(A)=\Phi 1 \quad\left(C_{*}(A)=\left\{c \in C(A) \mid c^{*}=c\right\}\right.$,

(iii) $C(A)=\Phi 1$ if $n \geq 2$,

(iv) $N(A)=\Phi 1$ if $n \geq 3$.

Proof. (i) $\operatorname{Comm}\left(A_{0}\right)=C\left(A_{0}\right)=A_{0}$ for $A_{0}$ of dimension 1 or 2 , so by (6.8) (viii)-(xii) this continues to hold for all $A$ built up from $A_{0}$. (ii)-(iii) hold by definition if $n=0(A=\Phi 1)$ or $n=1(A=\Omega)$; if true for $n-1$ and $n \geq 2$ then $A=\mathbf{C}(B)$ where $B$ of dimension $\geq 2$ contains cancellable $S k(b)$, so (6.8)(xii) shows $C(A)=C_{*}(B)=\Phi 1$ (by induction (ii)), and so $C(A)=C_{*}(A)=\Phi 1$ is true for $n$. (iv) If $n \geq 3$ then $A=\mathrm{C}(B)$ where $B$ of dimension $\geq 4$ contains cancellable commutators, hence (6.8)(xi) shows $N(A)=C(B)=\Phi 1$ (by (iii)).

6.10. REMARK. R. Erdmann [3] gave a lengthy proof that generalized Cayley-Dickson algebras over fields of characteristic $\neq 2$ contain no proper one-sided ideals. The above simple proof works for arbitrary scalars, and doesn't require starting from a Cayley algebra. Note that if commuting elements are central (as in all generalized Cayley-Dickson algebras, using (6.9)(i)) then the forbidden $m$ 's in (6.8)(vii) are precisely all central $\gamma$ with $\mu \gamma \gamma^{*}=1, \gamma[A, A]=0$, hence $\gamma$ invertible forces $[A, A]=0$ and $A$ is commutative. Thus the condition (vii) reduces to: $A$ is simple but not commutative with $\gamma \in C(A), \mu \gamma \gamma^{*}=1$ (the same as in the (vi) except that the involution may be nontrivial).

\section{REFERENCES}

[1] E. Becker, Über eine Klasse flexibler quadratischer Divisionsalgebren, J. für Reine u. Angew. Math., 256 (1972), 25-57.

[2] H. Braun and M. Koecher, Jordan-Algebren, Springer Verlag, Berlin, 1966.

[3] R. Erdmann, Über verallgemeinerte Cayley-Dickson Algebren, J. für Reine u. Angew. Math., 250 (197), 153-181.

[4] R. Kunze and S. Scheinberg, Algebras with scalar involution, to appear.

[5] H. Petersson and M. Racine, Jordan algebras of degree 3 and the Tits process, to appear.

[6] R. D. Schaefer, On the algebras formed by the Cayley-Dickson process, Amer. J. Math., 76 (1954), 435-446.

Received April 18, 1983

UNIVERSITY OF VIRGINIA

CharlotTesVille, VA 22903 



\section{PACIFIC JOURNAL OF MATHEMATICS \\ EDITORS}

Donald BABBITT (Managing Editor)

University of California

Los Angeles, CA 90024

J. Dugundu

University of Southern Californa

Los Angeles, CA 90089-1113

R. FINN

Stanford University

Stanford, CA 94305

HermanN FLaSChKa

University of Arizona

Tucson, AZ 85721

\author{
C. C. MOORE \\ University of California \\ Berkeley, CA 94720 \\ ARTHur Ogus \\ University of California \\ Berkeley, CA 94720 \\ Hugo Rossi \\ University of Utah \\ Salt Lake City, UT 84112 \\ H. SAMELSON \\ Stanford University \\ Stanford, CA 94305
}

ASSOCIATE EDITORS
R. ARENS
E. F. BECKENBACH
B. H. NeUmanN
F. WOLF
K. YOSHIDA (1906-1982)

\section{SUPPORTING INSTITUTIONS}

UNIVERSITY OF ARIZONA

UNIVERSITY OF BRITISH COLUMBIA

UNIVERSITY OF OREGON

CALIFORNIA INSTITUTE OF TECHNOLOGY

UNIVERSITY OF CALIFORNIA

MONTANA STATE UNIVERSITY

UNIVERSITY OF SOUTHERN CALIFORNIA

UNIVERSITY OF NEVADA, RENO

STANFORD UNIVERSITY

UNIVERSITY OF HAWAII

NEW MEXICO STATE UNIVERSITY

UNIVERSITY OF TOKYO

UNIVERSITY OF UTAH

WASHINGTON STATE UNIVERSITY

OREGON STATE UNIVERSITY

UNIVERSITY OF WASHINGTON 


\section{Pacific Journal of Mathematics}

\section{Vol. 116, No. $1 \quad$ November, 1985}

K. Adachi, Le problème de Lévi pour les fibrés grassmanniens et les variétés

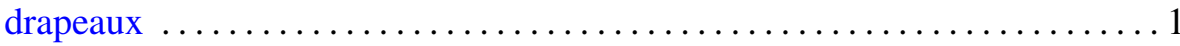

John MacLeod Ball, Remarks on the paper: "Basic calculus of variations" . . . 7 John Kelly Beem and Phillip E. Parker, Whitney stability of solvability . . . 11 Alberto Facchini, Decompositions of algebraically compact modules .......25

S. S. Khare, Finite group action and equivariant bordism $\ldots \ldots \ldots \ldots . \ldots 39$

Horst Leptin, A new kind of eigenfunction expansions on groups $\ldots \ldots \ldots . .45$

Pei-Kee Lin, Unconditional bases and fixed points of nonexpansive

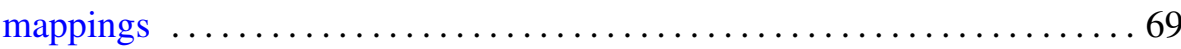

Charles Livingston, Stably irreducible surfaces in $S^{4} \ldots \ldots \ldots \ldots \ldots 77$

Kevin Mor McCrimmon, Nonassociative algebras with scalar involution . . .885

Albert Milani, Singular limits of quasilinear hyperbolic systems in a

bounded domain of $\mathbf{R}^{3}$ with applications to Maxwell's equations

Takemi Mizokami, On $M$-structures and strongly regularly stratifiable

spaces

Jesper M. Møller, On the homology of spaces of sections of complex

projective bundles

Nikolaos S. Papageorgiou, Carathéodory convex integrand operators and

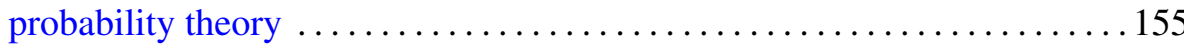

Robert John Piacenza, Transfer in generalized prestack cohomology 185

Lance W. Small and Adrian R. Wadsworth, Integrality of subrings of matrix rings ...

James Michael Wilson, On the atomic decomposition for Hardy spaces 